\title{
Design and Control of a Dual-Stage Disk Drive Servo System with a High-Aspect Ratio Electrostatic Microactuator
}

\author{
Kenn Oldham, Sarah Felix, Richard Conway, and Roberto Horowitz
}

\begin{abstract}
Dual-stage servo systems have been proposed as a method for attaining increased bit densities from computer hard disk drives. To aid in controller operation, the second-stage microactuator used in a dual-stage system should operate at low voltages, feature relative position sensing, and have dynamics that interact well with the dynamics of the original drive servo. This paper describes a high-aspect ratio electrostatic microactuator modified to improve dynamic interactions, relative position sensor performance, and device yield. A multi-rate, multivariable control design technique was used to evaluate the closedloop performance of a disk drive system with a microactuator and relative position sensing. Closed-loop simulations reveal that the proposed configuration can reduce off-track position error. Addressing both hardware design and control design, this paper demonstrates the success of an integrated mechatronic approach to hard disk drive servo control.
\end{abstract}

\section{INTRODUCTION}

The continual increase of bit densities in computer disk drives requires ever-improving performance from the mechanical systems within the drives. The data storage industry is currently targeting disk drive bit densities of 1 terabyte per square inch (Tbpsi). This will require read-write head positioning with $3 \sigma$ tracking errors of less than $5 \mathrm{~nm}$. A conventional disk drive is shown in Fig. 1. As the disk spins, it causes turbulent airflow to move past the servo arm, exciting the resonant vibrations of the E-block and flexible suspension that support the read-write head over the disk. At 1 Tbpsi tracking errors, this high frequency airflow-induced vibration is expected to become the dominant obstacle to accurate servo system tracking.

A potential solution to airflow-induced vibration of the servo arm is to install a second actuator at the tip of the servo arm. Such a microactuator can have a higher bandwidth than the conventional voice-coil motor (VCM) in the disk drive, and can be located beyond the region of suspension vibration. Various disk drive dual-stage servo configurations place a microactuator in the flexible suspension [1] [2] [3] [4], between the suspension and slider containing the readwrite head [5] [6] [7] [8], or inside the slider itself [9] [10]. These arrangements provide increasing bandwidth from the microactuator in exchange for increasing design and processing complexity. Microactuators described in the literature

This work was supported in part by National Science Foundation grant CMS-0428917, the Information Storage Industry Consortium and the Computer Mechanics Laboratory at UC Berkeley

$\mathrm{K}$. Oldham is with the department of Mechanical Engineering at the University of Michigan. oldhameumich.edu

S. Felix, R. Conway, and R. Horowitz are with the Department of Mechanical Engineering at the University of California, Berkeley. rconway345@berkeley.edu, sarfelix@me.berkeley.edu, horowitz@me.berkeley.edu

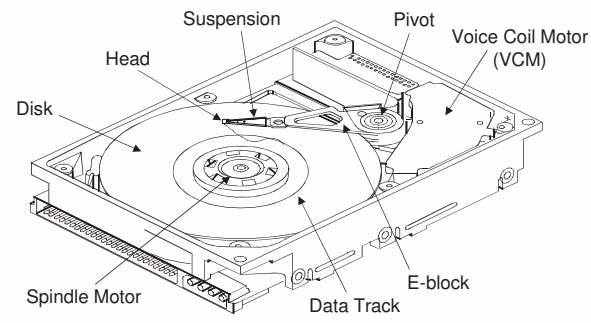

Fig. 1. Mechanical components of a typical hard disk drive

typically suffer from excessive voltage requirements and undesirable interactions between the dynamics of the actuator and of the servo arm when the actuator is installed. Previously reported designs have also tended to focus exclusively on actuation performance, neglecting other capabilities useful in a dual-stage system, such as relative-position sensing and high-conductivity interconnects.

The microactuator described in this paper incorporates many useful features that improve dual-stage servo performance. Deep-trench isolation and deep-reactive ion etching are used to provide high-force, low-voltage actuation, as described in previous articles. A novel flexure configuration allows in-plane and out-of-plane stiffness to be tailored individually to balance requirements on microactuator dynamics during read-write head flight. The microactuator includes dedicated capacitive sensing arrays for high-resolution relative position sensing, and the microactuator fabrication process has been revised to greatly improve interconnect quality and sensor performance. Completed microactuators have been successfully installed on multi-piece suspensions (Fig. 2) with improved vibration behavior over previous models, permitting more aggressive dual-stage controller designs.

Finally, complete system models were obtained based on experimental responses of the microactuator and capacitive sensor. The models were used to design a multi-inputmulti-output (MIMO), multi-rate controller. The controller synthesis incorporated several constraints that reflect physical limitations of the microactuator and relative position sensing limitations. Closed-loop simulations provided insight into how the device design affects closed-loop performance.

\section{MICROACTUATOR DESIGN}

The high-aspect ratio microactuator consists of a central shuttle holding a pico slider and read-write head, driven 
TABLE I

NOMINAL MICROACTUATOR DESIGN PARAMETERS AND EXPECTED PERFORMANCE

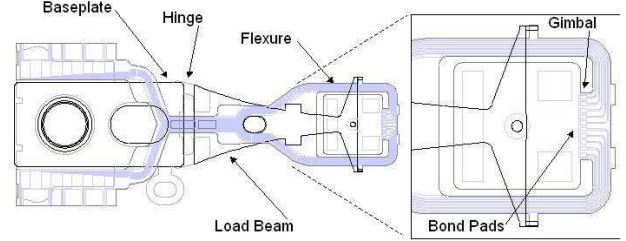

Fig. 2. $15 \mathrm{~mm}$ MEMS-ready suspension

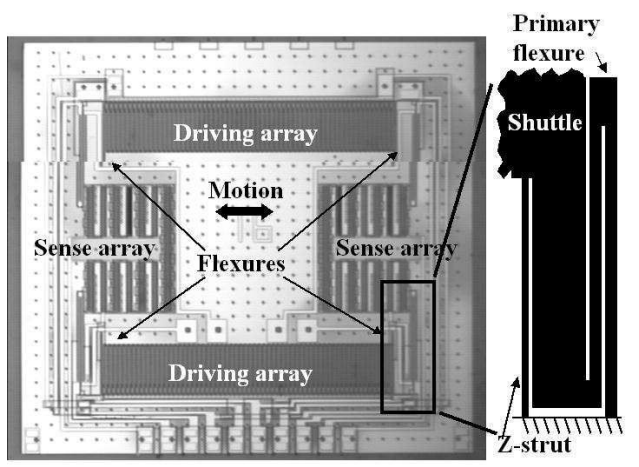

Fig. 3. Full microactuator layout, with hybrid flexure design

by gap-closing electrostatic parallel-plate fingers. A view of the microactuator structure is shown in Fig. 3. Differential parallel-plate arrays are interlaced using deep-trench isolation. This feature along with high-aspect ratio trenches produced by deep reactive-ion etching (DRIE) result in excellent force generation, as has been described in previous publications [11] [12]. The differential configuration results in an actuation force that is linear to within $5 \%$ over a $1 \mu \mathrm{m}$ stroke length. The linearized actuation force, $F$, is given by the equation

$$
F=2 \epsilon V V_{b} \frac{t L_{f}}{g_{0}^{2}} N_{f}
$$

where $\epsilon$ is the permittivity of air, $V$ is the applied input voltage, $V_{b}$ is a DC bias voltage on the shuttle fingers, $t$ is the device thickness, $L_{f}$ is the driving finger length, $g_{0}$ is the nominal gap between fingers, and $N_{f}$ is the number of driving fingers.

Four pairs of flexures support the moving shuttle, with one pair located at each corner. Each flexure pair includes a long, wide main spring and a narrow z-strut, illustrated in Fig. 3. The main spring provides space for electrical interconnects and provides the bulk of resistance to in-plane motion, while the high-aspect ratio z-struts add significant out-ofplane stiffness with only a minor effect on in-plane motion. This allows the natural frequency of the microactuator to be precisely selected as best suited for closed-loop operation.

Sets of capacitive comb finger arrays act as position

\begin{tabular}{|c|c|c|}
\hline Parameter (units) & Label & Value \\
\hline \hline Device thickness $(\mu \mathrm{m})$ & $t$ & 100 \\
\hline Mass of shuttle and actuator $(\mathrm{mg})$ & $m_{t}$ ot & 2.2 \\
\hline Trench widths $(\mu \mathrm{m})$ & $g_{0}$ & 4.0 \\
\hline Number of actuator fingers & $N_{f}$ & 126 \\
\hline Actuator finger length $(\mu \mathrm{m})$ & $L_{f}$ & 282 \\
\hline Main spring width $(\mu \mathrm{m})$ & $w_{k}$ & 16 \\
\hline Total spring constant $(\mathrm{N} / \mathrm{m})$ & $k_{t}$ ot & 497 \\
\hline Natural frequency $(\mathrm{Hz})$ & $\omega_{n}$ & 2140 \\
\hline Bias Voltage $(\mathrm{V})$ & $V_{b}$ & 15 \\
\hline Force/Volt $(\mu \mathrm{N} / \mathrm{V})$ & $\mathrm{F} / \mathrm{V}$ & 77 \\
\hline Displacement/Volt $(\mathrm{nm} / \mathrm{V})$ & $\mathrm{x} / \mathrm{V}$ & 81 \\
\hline Number of sensor fingers & $N_{s}$ & 304 \\
\hline Sensor finger length $(\mu \mathrm{m})$ & $L_{s}$ & 40 \\
\hline Sensor capacitance $(\mathrm{pF})$ & $C_{s}$ & 2.7 \\
\hline
\end{tabular}

sensors to measure shuttle displacement relative to the tip of the disk drive suspension, known as the relative position error signal (RPES). The use of the RPES in dual-stage control can improve closed-loop performance by permitting damping of microactuator resonant modes and preventing interference between VCM and microactuator motion [13]. The capacitive sensors are operated differentially, and the change in capacitance, $\Delta C_{s}$, with displacement, $\Delta x$ is given simply by the formula:

$$
\frac{\Delta C_{s}}{\Delta x}=\epsilon_{0} \frac{t}{g_{0}} N_{s}
$$

where $N_{s}$ is the number of sensor fingers.

Key microactuator design parameters and anticipated properties are listed in Table I.

\section{MICROACTUATOR FABRICATION}

Fig. 4 shows the process flow for microactuator fabrication. Microactuators were fabricated from silicon-oninsulator wafers with a $100 \mu \mathrm{m}$ thick device layer. Wafers were coated with highly-doped polysilicon to increase conductivity of vias and sensor electrodes. Deep-trench isolation trenches were defined by lithography and etched by DRIE (Fig. 4a). For electrical insulation, the trenches are coated by a thin $0.25 \mu \mathrm{m}$ silicon nitride film deposited by Low Pressure Chemical Vapor Deposition (LPCVD). The remainder of the trenches were refilled with undoped LPCVD polysilicon (Fig. 4b). Effective refill and planarization of the deep isolation trenches is critical to device quality. For the highaspect ration trenches a brief reactive ion-etch (RIE) was performed to etch the polysilicon film back to the nitride surface, with an overetch to expose shallow keyholes. A second polysilicon deposition refilled these keyholes, leaving a thinner layer of excess polysilicon on the wafer surface. This allowed more precise control of a second etch-back and planarization step by chemical mechanical polishing (CMP). After polishing, a final $0.2 \mu \mathrm{m}$ LPCVD silicon nitride layer was deposited, completing the isolation trenches (Fig. 4c). 


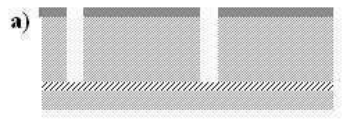

b)
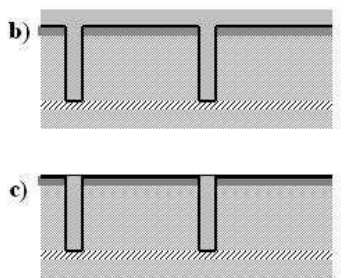

d)
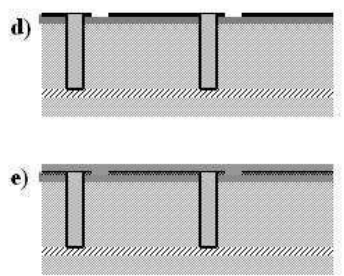

Single crystal silicon

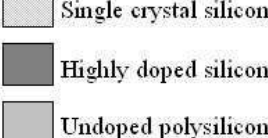

Undoped polysilicon
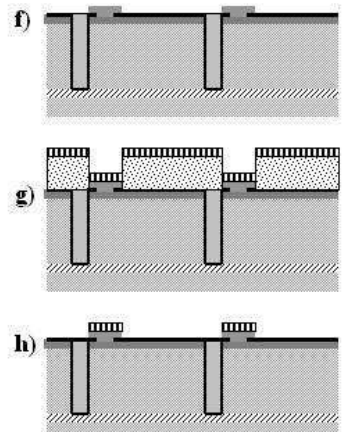

i)
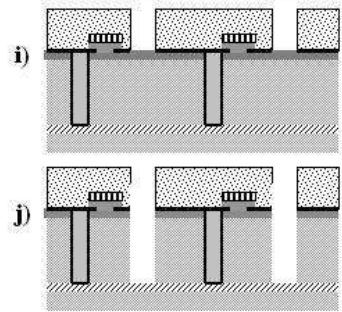

Silicon nitride

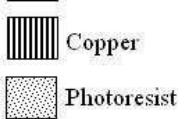

Fig. 4. High-aspect ratio microactuator process flow

The CMP process provided an excellent surface for interconnect formation. Contact holes were patterned by lithography and etched through the silicon nitride film by RIE (Fig. 4d). Interconnects consisted of a silicon-metal stack to provide mechanical robustness and high conductivity. A highly doped polysilicon film was patterned by lithography and etched by RIE (Fig. 4f). A chrome adhesion layer and $1000 \AA$-thick copper film are then evaporated onto a $9 \mu \mathrm{m}$ thick, patterned, photoresist layer, to form the upper layer of the interconnects in a lift-off procedure (Fig. 4g,h). The copper layer provided improved conductivity and, due to its resistance to hydrofluoric acid, permitted a dramatically simplified release procedure from previous microactuator fabrication processes.

A final lithography step and DRIE step were performed to define the electrostatic fingers and other features of the completed microactuator. During this final lithography step, a $9 \mu \mathrm{m}$-thick photoresist film was again necessary in order to encapsulate the metallized interconnects (Fig. 4i,k). The trench width targeted for this step was $4 \mu \mathrm{m}$, but erosion of trench sidewalls during the etching process resulted in actual trench widths as large as $8 \mu \mathrm{m}$. This proved to be a significant limitation on microactuator performance, as will be discussed in Section V. Microactuators were released from the wafer by breaking the tethers supporting the devices and immersing the dies in hydrofluoric acid (HF). The thick photoresist layer was dissolved in acetone, and microactuator surfaces were cleaned with $\mathrm{O} 2$ and SF6 plasmas.

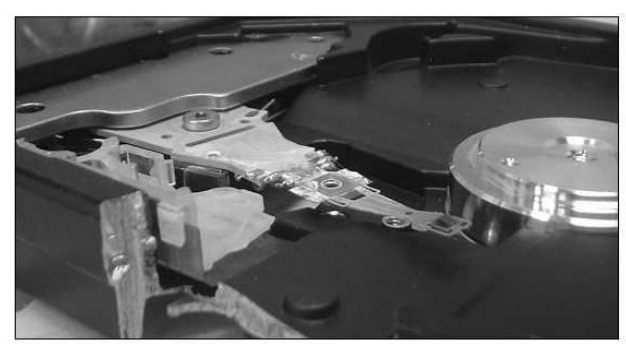

Fig. 5. Assembled dual-stage test system

\section{OPEN-LOOP EXPERIMENTAL RESULTS}

Finished microactuators were installed on $15 \mathrm{~mm}$ MEMS ready suspensions provided by Hutchinson Technology, Inc. Off-track vibration modes for these suspensions were expected to exceed $4 \mathrm{kHz}$ in frequency, providing a wide separation from the natural frequency of the microactuator. This separation of modes improves dual-stage servo capabilities compared to microactuators installed on earlier, longer disk drive suspensions.

Microactuators were aligned to sliders and suspensions using bulk-micromachined silicon fixtures on a 3-DOF positioning stage. These fixtures were intended to reduce unwanted dynamics in the disk drive due to inaccuracies of the microactuator installation process. A slider was attached to a microactuator and the microactuator to a suspension using epoxy, after which ultrastonic wire bonding was used to create electrical connections between the suspension and microactuator. The suspension assembly was then glued to a modified disk drive E-block, with a slight bend at the tip to reduce slider pitch and metal strips attached to provide connections for electrical leads from the suspension. Finally, ultrasonic wire bonding was used to connect these metal strips and leads.

The assembled servo arm was installed in a commercial disk drive as shown in Fig. 5 with the disk removed. Displacement of the read-write head and suspension tip were measured using a Laser Doppler Velocimeter (LDV). A 2.5 $\mathrm{cm}$ disk was used to improve access and signal quality from the LDV. Rotation speed of the disk was $7200 \mathrm{rpm}$.

Fig. 6 shows the transfer function from microactuator input to read-write head displacement during disk drive operation. The microactuator has a natural frequency of $1780 \mathrm{~Hz}$ and a static gain of $17 \mu \mathrm{N} / \mathrm{V}$. The natural frequency is located well away from suspension vibration modes occuring above $3.5 \mathrm{kHz}$. The actuation force meets or exceeds that of other published results for an actuated slider, despite trench erosion that widens that gaps betweens electrodes. The oversized trench width limits the performance of the actuator, as shown in Table II, but the experimental gains are still adequate for vibration control.

Fig. 7 shows the transfer function from VCM input to displacement of the suspension tip and the read-write head. The microactuator's resonant peak is visible between a low frequency vibration mode associated with the servo arm flex cable, and the higher frequency suspension vibration modes. 


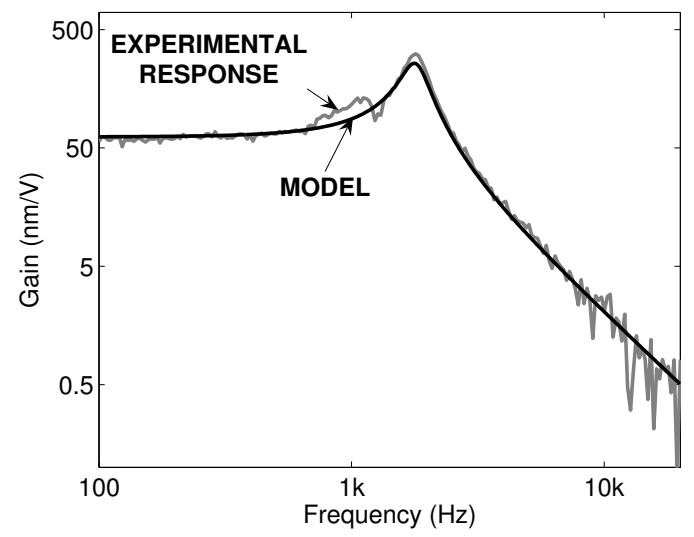

Fig. 6. Experimental frequency response of high-aspect ratio microactuator during flight

TABLE II

THEORETICAL MICROACTUATOR PERFORMANCE WITHOUT AND WITH TRENCH EROSION VERSUS EXPERIMENTAL RESULTS

\begin{tabular}{|c|c|c|c|}
\hline & $\begin{array}{c}\text { Theoretical } \\
\text { performance, } \\
\text { nominal } \\
\text { trench width }\end{array}$ & $\begin{array}{c}\text { Theoretical } \\
\text { performance, } \\
\text { actual } \\
\text { trench width }\end{array}$ & $\begin{array}{c}\text { Experimental } \\
\text { performance }\end{array}$ \\
\hline \hline Gap width $(\mu \mathrm{m})$ & 4.0 & 7.6 & 7.6 \\
\hline Natural frequency $(\mathrm{Hz})$ & 2140 & 1600 & 1780 \\
\hline Static gain $(\mu \mathrm{N} / \mathrm{V})$ & 77 & 18 & 17 \\
\hline Static gain $(\mathrm{nm} / \mathrm{V})$ & 195 & 82 & 62 \\
\hline Damping ratio & & \multicolumn{2}{|l}{} \\
\hline
\end{tabular}

It is clear that the microactuator dynamics attenuate head vibration even in open-loop, as the microactuator acts as a low pass filter between the suspension dynamics and read write head. However, the microactuator's own resonance may be excited by airflow, motivating the addition of relative position error sensing.

The RPES was implemented using a fully differential capacitive sensing circuit with high-frequency modulation, as shown in the schematic in Fig. 8. The sensing circuit used conventional high frequency multiplexing of the sensor input voltage to reduce noise and low frequency disturbance. A 3.3 $\mathrm{V}, 400 \mathrm{kHz}$ square wave was used as the input voltage and modulation signal. The output low-pass filter was followed by a high-pass filter to form a pass band pass between $700 \mathrm{~Hz}$ and $4 \mathrm{kHz}$, in order to focus on the resonant peak of the microactuator, where disturbances to the microactuator motion result in the most significant errors in read-write head motion.

Fig. 9 shows the transfer function from microactuator input to sensor output during disk drive operation, along with a scaled view of the bandpass filter to show the underlying circuit dynamics. The resonant peak of the microactuator is plainly visible, even for relatively small microactuator motions. Sensor resolution is limited by high frequency disturbances and feedthrough distortions from the driving



Fig. 7. Experimental transfer functions from VCM input to read-write head (dashed) and to suspension tip (gray line) displacement. The model of the VCM to suspension tip transfer function is shown in black.

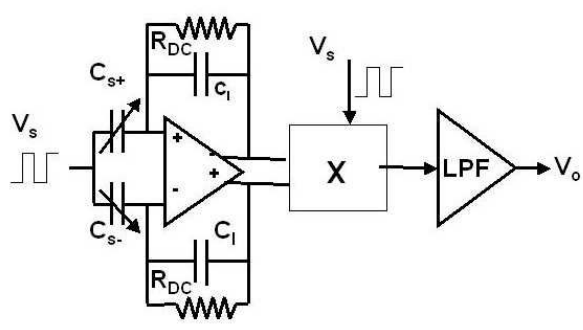

Fig. 8. Schematic view of relative position error sensing (RPES) circuit

signal. The most significant high frequency "noise" source was ringing at the modulation frequency due to unmodeled mismatches in input resistance and parasitic capacitances between the two differential sensing capacitors. Feedthrough is the primary limitation on the resolution of capacitive sensing in many MEMS devices. While feedthrough in the proposed design is reduced by the low driving voltage of the microactuator and separate driving and sensing arrays, it is still a significant source of measurement error.

In tests of the microactuator alone, $3 \mathrm{~nm}$ sensor resolution in the presence of sensor noise and a worst case feedthrough distortion of $6 \mathrm{~nm}$ were achieved. After installation, microactuator resolution is degraded to approximately $17 \mathrm{~nm}$ when measuring external "disturbances," such as motion excited by the VCM. Feedthrough and associated ringing due to microactuator input voltages cause even greater errors, with $40 \mathrm{~nm}$ resolution and $19 \mathrm{~nm}$ distortion due to feedthrough. The degradation in performance after installation is attributed to longer leads and larger parasitic capacitances when the suspension, E-block, and additional cables are placed between the microactuator and sensing circuitry. Ideally, sensing circuitry would be placed on the E-block itself to improve sensor performance. A more aggressive bandpass filter or controller-based model of feedthrough behavior could also potentially improve sensor resolution. 


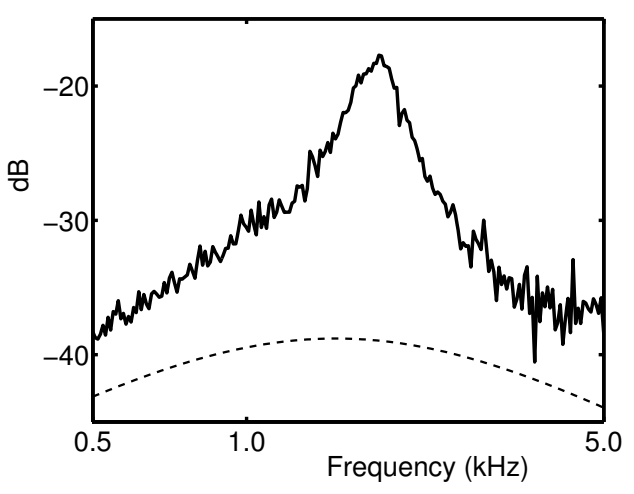

Fig. 9. Transfer function from microactuator input to microactuator displacement (solid line), measured by the RPES circuit during flight. Also shown is the circuit bandpass filter (dashed line), not to scale.

\section{CLOSED-LOOP CONTROL DESIGN AND SIMULATION}

To evaluate the benefit of microactuator improvements and relative position sensing for tracking control, a closed-loop controller was designed and simulated. The control design strategy was based on optimal multi-rate, multivariable control synthesis for dual stage hard disk drives described in [14]. Fig. 10 illustrated the feedback representation for an optimal MIMO controller, called a linear fraction transformation (LFT). $S$ and $H$ represent the multi-rate sample and hold, respectively. $K$ denotes the optimal discretetime controller. For the general optimal control problem, a controller is designed to minimize some norm, $T_{z w}$, between the disturbances, $w$, and the outputs, $z$. The system is multirate since the capacitive sensing and actuators can operate at a higher rate than the fixed rate of the conventional position error signal (PES). In this paper, the sampling rate of the PES was $75 \mathrm{kHz}$, while the RPES sampling rate and the actuation rates were $150 \mathrm{kHz}$. [13] showed that this control scheme can achieve superior tracking performance compared to both single rate and single-input-single-output strategies.

The control design used in this study is different from that described in [14] in several ways. First, the control design considers $\mathrm{H}_{2}$ control instead of mixed $\mathrm{H}_{2} / \mathrm{H}_{\text {inf }}$. For $H_{2}$ control, $T_{z w}$ represents a 2-norm mapping, so it is a more natural formulation for minimizing the variance of the PES. Second, the control synthesis is based on a nominal plant, ignoring robustness. This is useful for evaluating the performance of a given hardware configuration. Third, the constraints in the minimization are formulated differently. Here, explicit constraints are imposed on certain signals such as control effort, while the PES is directly minimized. This can be stated as follows:

$$
\begin{aligned}
\left\|T_{R P E S, w}\right\|_{2}^{2} & <\gamma_{1} \\
\min _{K}\left\|T_{P E S, w}\right\|_{2}^{2} \quad \text { subject to } \quad\left\|T_{u_{v}, w}\right\|_{2}^{2} & <\gamma_{2}, \\
\left\|T_{u_{m}, w}\right\|_{2}^{2} & <\gamma_{3}
\end{aligned}
$$

where $\gamma_{i}$ is the constraint for the corresponding norm. This is in contrast to minimizing a norm that reflects a weighted

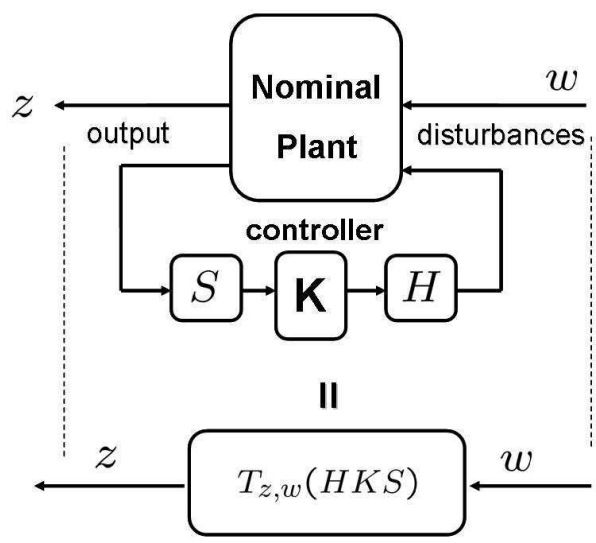

Fig. 10. Feedback representation of optimal MIMO multirate controller

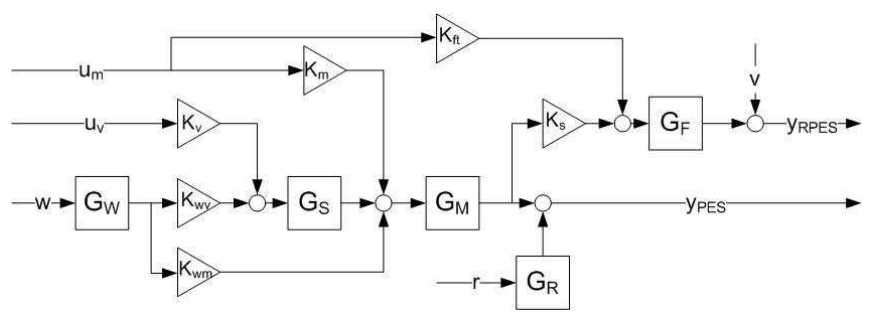

Fig. 11. Block diagram of plant model

combination of the PES along with the other signals:

$$
\begin{aligned}
\min _{K}\left\|T_{z, w}\right\|_{2}^{2}=\min _{K}\left\{\left\|T_{P E S, w}\right\|_{2}^{2}+\alpha_{1}\left\|T_{R P E S, w}\right\|_{2}^{2}\right. \\
\left.+\alpha_{2}\left\|T_{u_{v}, w}\right\|_{2}^{2}+\alpha_{3}\left\|T_{u_{m}, w}\right\|_{2}^{2}\right\},
\end{aligned}
$$

where $\alpha_{i}$ is a weights for the corresponding norm. As will be explained in the following, this turns out to be a useful tool for device design.

\section{A. Plant Model}

The complete system model for control design incorporated experimental results described in previous sections. Fig. 11 shows a block diagram of the plant model. The VCM and microactuator inputs are $u_{v}$ and $u_{m}$, respectively. $y_{P E S}$ and $y_{R P E S}$ are the measurements corresponding to the subscripts. The remaining labels in the diagram will be described in the following.

The microactuator mode was modeled as a second order transfer function, with the natural frequency, $\omega_{M}$, and damping ratio, $\zeta_{M}$, estimated from the response shown in Fig. 6. The transfer function is

$$
G_{M}(s)=\frac{1}{s^{2}+\zeta_{M} \omega_{M} s+\omega_{M}^{2}} .
$$

The model is shown in Fig. 6 along with the experimental results. Similarly, the suspension dynamics were modeled as a summation of $N$ second order modes, with some modes neglected to keep model order manageable. The transfer function is given by 
TABLE III



Fig. 12. Bode diagram of $G_{w}$ representing the frequency content of the windage disturbance

$$
G_{S}(s)=\sum_{i=1}^{N} \frac{A_{s i}}{s^{2}+\zeta_{s i} \omega_{s i} s+\omega_{s i}^{2}},
$$

where $A_{s i}, \zeta_{s i}$, and $\omega_{s i}$ are respectively the modal constant, damping ratio and natural freqency of the $i$ th mode. The model response is shown in Fig. 7. The suspension modes were excited by both the VCM and by windage. The gain vectors $K_{v}, K_{w v}$, captured the appropriate magnitude of these excitations, respectively. The microactuator dynamics were excited by suspension tip motion (the output of $G_{s}$ ), microactuator input, and windage.

The capacitive sensor was modeled with the following filter to reflect the circuit dynamics:

$$
G_{F}(s)=K_{F}^{2} *\left(\frac{\omega_{L}}{s+\omega_{L}}\right)^{2}\left(\frac{\frac{1}{\omega_{H}} s}{\frac{1}{\omega_{H}} s+1}\right)^{2},
$$

where $K_{F}, \omega_{H}$, and $\omega_{L}$ are the filter gain, high pass cutoff frequency, and low pass cutoff frequency respectively. Sensor gain $K_{S}$ represents the conversion of displacement to voltage. RPES sensor noise, represented by $v$ in the block diagram, was assumed white with a Gaussian distribution and a variance reflecting the $40 \mathrm{~nm}$ resolution observed in the experimental results. In addition, feedthrough distortion was modeled by sending $u_{m}$ through a gain, $F_{f t}$, and adding this to the input to the sensor tranfer function, $G_{F}(s)$.

The system was subject to several exogenous input disturbances which are considered in $H_{2}$ control. Track runout, which consists of low frequency repeatable and nonrepeatable disturbances, was modeled as white noise, $r$, filtered by a transfer function, $G_{R}(s) . G_{R}(s)$ was obtained from [13]. Windage was modeled as white noise, $w$, filtered by the transfer function $G_{W}(s)$, which was estimated from experimental data and is shown in Fig. 12. Table III summarizes values of various model parameters.
MODEL PARAMETERS

\begin{tabular}{|c|c|c|}
\hline Parameter (units) & Label & Value \\
\hline Filter Gain & $K_{F}$ & 1.2 \\
\hline Low Pass Cutoff Frequency (Hz) & $\omega_{L}$ & $4.2 \mathrm{e} 3$ \\
\hline High Pass Cutoff Frequency (Hz) & $\omega_{H}$ & $2.3 \mathrm{e} 4$ \\
\hline Sensor Gain (V/nm) & $K_{S}$ & $5 \mathrm{e} 5$ \\
\hline Feedthrough Gain (V/V) & $K_{f t}$ & $18 \mathrm{e}-9 * K_{S}$ \\
\hline Sensor Noise Variance V' & $v$ & $\left(40 e-9 * K_{S}\right)^{2}$ \\
\hline Runout Noise Variance & $r$ & 1 \\
\hline
\end{tabular}

\section{B. Control Design and Simulation}

As mentioned above, the control synthesis incorporates constraints on certain quantities that can reflect hardware limitations. Specifically, the VCM input voltage, $u_{v}$, and microactuator voltage, $u_{m}$, as well as the RPES, are constrained. There were two considerations in setting the constraints: physical limitations and sensing limitations. The physical limitations of $u_{v}$ and $u_{m}$ are based on range of device operation and hardware experience. The physical limitation of the RPES is the displacement range of the capacitive arrays which avoids electrostatic pull-in. However, the previous section described limitations of the capacitive sensing circuit that reduce the range of meaningful measurements. Sensing limitations can be simulated by imposing more strict constraints to ensure that this meaningful measurement range is not exceeded. Specifically, the actual RPES range can be narrowed, and $u_{m}$ can be limited to avoid excessive feedthrough distortion.

For comparison, the control design was performed on the improved system with and without capacitive sensing, as well as an earlier model with older hardware [13] and no capacitive sensing. The target for tracking performance was $5 \mathrm{~nm}$ at $3 \sigma$.

First, controllers were designed for the old and new systems using physical constraints. With the multi-rate $\mathrm{H}_{2}$ controller, the older design was able to achieve $5.4 \mathrm{~nm}$ tracking, which falls short of the target. However, the new system without capacitive sensing achieved $4.6 \mathrm{~nm}$. This demonstrates that the hardware improvements alone were beneficial for tracking performance. Table IV summarizes the $3 \sigma$ values of the various signals and their maximum allowed values.

Next, capacitive sensing was introduced into the model with the new hardware. Constraints on RPES and $u_{m}$ were tightened to reflect sensing limitations. As such, the closedloop system was again only able to achieve $5.4 \mathrm{~nm}$. Tracking performance suffered due to imposing tighter limitations on the microactuator effort. To evaluate just how much microactuator effort would be needed along with capacitive sensing to recover the $4.6 \mathrm{~nm}$ result, the next iteration was performed in which the PES signal is constrained to 4.6 $\mathrm{nm}$ and $u_{m}$ is minimized. The resulting $3 \sigma$ value of $u_{m}$ (shown in Table IV) increases to its original value near the upper bound of 20V. This indicates that acceptable tracking is possible provided improvements can be made to the sensor 
TABLE IV

NOMINAL CLOSED-LOOP CONTROL SIMULATION RESULTS ( $3 \sigma$ VALUES)

\begin{tabular}{|l|l|l|l|l|}
\hline Plant (Controller Order) & $\begin{array}{l}\text { PES } \\
(\mathrm{nm})\end{array}$ & $\begin{array}{l}\text { RPES } \\
(\mathrm{nm})\end{array}$ & $\begin{array}{l}u_{v} \\
(\mathrm{~V})\end{array}$ & $\begin{array}{l}u_{m} \\
(\mathrm{~V})\end{array}$ \\
\hline 1-Old plant, no RPES (11) & 5.4 & 45.9 & 2.2 & 20.0 \\
\hline 2-New plant, no RPES (15) & 4.6 & 37.8 & 4.9 & 20.0 \\
\hline Maximum allowed & & $\mathbf{1 0 0 0}$ & $\mathbf{5}$ & $\mathbf{2 0}$ \\
\hline \hline 3-New plant, with RPES (19) & 5.4 & 47.2 & 3.4 & 5.0 \\
\hline Maximum allowed & & $\mathbf{1 0 0}$ & $\mathbf{5}$ & $\mathbf{5}$ \\
\hline \hline 4-New plant, with RPES (19) & 4.6 & 38.3 & 5.0 & 20.0 \\
\hline Maximum allowed & $\mathbf{4 . 6}$ & $\mathbf{1 0 0}$ & $\mathbf{5}$ & \\
\hline
\end{tabular}

TABLE V

Closed-Loop CONTROL SimUlations With PERTURBed PLANT

\begin{tabular}{|l|l|l|l|l|}
\hline Controller & $\begin{array}{l}\text { PES } \\
(\mathrm{nm})\end{array}$ & $\begin{array}{l}\text { RPES } \\
(\mathrm{nm})\end{array}$ & $\begin{array}{l}u_{v} \\
(\mathrm{~V})\end{array}$ & $\begin{array}{l}u_{m} \\
(\mathrm{~V})\end{array}$ \\
\hline 2-no RPES & 7.3 & 104.3 & 6.8 & 31.2 \\
\hline 4-with RPES & 5.3 & 58.1 & 4.9 & 23.0 \\
\hline Target/Limit & $\mathbf{5}$ & $\mathbf{1 0 0}$ & $\mathbf{5}$ & $\mathbf{2 0}$ \\
\hline
\end{tabular}

measurement circuitry in the presence of higher microactuator driving voltages. Improving microactuator gain with narrower trenches would allow more microactuator control at lower voltages, further alleviating the feedthrough limits on sensing. Note that along with improved tracking performance comes a higher controller order, summarized in Table IV, which is the result of greater complexity in the plant model.

Comparing the results of the second and fourth test, the RPES has little affect on nominal performance. This is because if the microactuator resonance can be exactly modeled, then the controller can compensate for it without additional sensing. As a final study, the controllers designed in the second and fourth test were connected to a plant model with $8 \%$ variation in the microactuator resonance frequency. The results from this closed-loop simulation are shown in Table V. Without the RPES, the PES variance is dramatically higher than in the nominal case, while $u_{v}$ and $u_{m}$ variances greatly exceed their limits. With RPES incorporated, the increase in the variances from the nominal case lessens. $u_{m}$ still exceeds its limit, emphasizing the need to improve trench width of the actuation arrays. It is thus demonstrated that the RPES is critical for robustness against plant variations.

\section{CONCLUSIONS AND FUTURE WORK}

This paper presents an integrated mechatronic approach to hardware and control design for a hard disk drive servo system. It described several improvements to a previouslyproposed dual-stage hard disk drive system using an electrostatic microactuator as the second stage input. Copper metallization of device interconnects improved conductivity and device yield. A new suspension geometry and flexure design allowed the microactuator resonance to be separated from the higher-frequency suspension vibration modes, a desirable feature for control. Finally, circuitry was developed to extract measurements from dedicated sensing arrays on the device, thus providing a relative position error signal.
Closed-loop simulations with a multi-rate, multivariable controller demonstrated that these hardware improvements were beneficial for tracking performance in a dual-stage hard disk drive. Further, the constrained $H_{2}$ control synthesis technique described proved to be a useful tool to evaluate hardware design with respect to closed-loop performance. It revealed that improvements to the capacitive sensing and microactuator trench width are critical for acceptable performance and robustness of the full system.

We are working on formulating a similar constrained synthesis algorithm for the robust $H_{2}$ control problem. This will be useful to further evaluate variations in device parameters with respect to closed-loop performance.

\section{ACKNOWLEDGMENTS}

The authors gratefully acknowledge Hutchinson Technology for providing the MEMS-ready suspension prototypes.

\section{REFERENCES}

[1] R. Evans, J. Griesbach, and W. Messner, "Piezoelectric microactuator for dual-stage control," IEEE Transactionson Magnetics, vol. 35, pp. 977-81, Mar. 1999.

[2] M. Tokuyama, T. Shimizu, H. Masuda, S. Nakamura, M. Hanya, O. Iriuchijima, and J. Soga, "Development of $\phi$-shaped actuated suspension for 100-ktpi hard disk drives," IEEE Transactions on Magnetics, vol. 33, no. 3, pp. 1184-6, Sept. 2001.

[3] I. Naniwa, S. Nakamura, S. Saegusa, and K. Sato, "Low voltage driven piggy-back actuator of hard disk drives," in IEEE International MEMS 99 Conference, Orlando, FL, USA, 1999, pp. 49-52.

[4] S. Koganezawa, K. Takaishi, Y. Mizoshita, Y. Uematsu, and T. Yamada, "Development of integrated piggyback milli-actuator for high density magnetic recording," in International Conference on Micromechatronics for Information and Precision Equipment, 1997, pp. 2023.

[5] D. Horsley, Ph.D. Dissertation: Microfabricated electrostatic actuators for magnetic disk drives. Berkeley, CA, USA: University of California, 1998.

[6] T. Hirano, L. Fan, T. Semba, W. Lee, J. Hong, S. Pattanaik, P. Webb, W.-H. Juan, and S. Chan, "High-bandwidth hdd tracking servo by a moving-slider micro-actuator," IEEE Transactions on Magnetics, vol. 35, pp. 3670-72, september 1999.

[7] H. Fujita, K. Suzuki, M. Ataka, and S. Nakamura, "A microactuator for head positioning system of hard disk drives," IEEE Transactionson Magnetics, vol. 35, pp. 1006-10, March 1999.

[8] H. Kuwajima and K. Matsuoka, "Thin film piezoelectric dual-stage actuator for hdd," in InterMag Europe, Session BSO4, Amsterdam, Netherlands, Apr. 2002, p. BS4.

[9] S. Nakamura, K. Suzuki, M. Ataka, and K. Mukasa, "An electrostatic microactuator for a magnetic head tracking system of hard disk drives," in Advances in Information Storage Systems, vol. 10, Singapore, Singapore, 1998, pp. 83-99.

[10] H. Toshiyoshi, M. Mita, and H. Fujita, "A mems piggyback actuator for hard-disk drives," Journal of Microelectromechanical Systems, vol. 11, no. 6, pp. 648-54, Dec. 2002.

[11] K. Oldham, X. Huang, and R. Horowitz, "Design, fabrication and control of a high-aspect ratio microactuator for vibration suppression in a hard disk drive," in Proceedings of the 16th IFAC World Congress, Prague, Czech Republic, 2005.

[12] T.-L. Chen, Y. Li, K. Oldham, and R. Horowitz, "Mems applications in computer disk drive dual-stage servo systems," Journal of the Society of Instrument and Control Engineers, vol. 41, no. 6, pp. 412-20, 2002.

[13] X. Huang, R. Nagamune, and R. Horowitz, "A comparison of multirate robust track-following control synthesis techniques for dual-stage and multi-sensing servo systems in hard disk drives," IEEE Transactions on Magnetic Recording, vol. 42, no. 7, pp. 1896-904, July 2006.

[14] H. X. Nagamune, R. and R. Horowitz, "Multirate track-following control with robust stability for a dual-stage multi-sensing servo system in hdds," in Proceedings of the 44th IEEE Conferenct on Decision and Control, 2005, pp. 3886-91. 